\title{
ANÁLISIS NUMÉRICO DEL COMPORTAMIENTO DEL AIRE EN UN SISTEMA DE DISTRIBUCIÓN DE AIRE ACONDICIONADO EMPLEANDO LOS MODELOS DE TURBULENCIA k- $\varepsilon$, RNG k- $\varepsilon$ Y EL MODELO DE LAS TENSIONES DE REYNOLDS
}

\author{
NUMERICAL ANALYSIS OF AIR BEHAVIOR IN AN AIR CONDITIONING \\ DISTRIBUTION SYSTEM USING k- $\varepsilon$ TURBULENCE, RNG k- $\varepsilon$ AND REYNOLDS \\ TENSIONS METHODS
}

\author{
Luz Rodríguez Collado $^{1} \quad$ María Collado Contreras $^{1} \quad$ Edgar Rodríguez Malaver $^{2} \quad$ Luis Patiño $^{2}$
}

Recibido 20 de marzo de 2007, aceptado 25 de febrero de 2008

Received: March 20, 2007 Accepted: February 25, 2008

\begin{abstract}
RESUMEN
En la presente investigación se empleó el método de los volúmenes finitos para simular numéricamente el comportamiento termofluidodinámico del aire en un sistema de distribución de aire acondicionado. Se describió el modelo matemático que rige el comportamiento del flujo de aire en el conducto de distribución y el sistema de ecuaciones obtenido fue cerrado mediante la aplicación un modelo de turbulencia o cierre: para ello se emplearon de forma individual el modelo k- $\varepsilon$, el modelo RNG k- $\varepsilon$ y el modelo de las tensiones de Reynolds. Fueron simulados tres casos de estudio y los resultados obtenidos de esas simulaciones indican que el modelo k- $\varepsilon$ presenta un mejor comportamiento numérico en el problema simulado, generando menores residuos en las variables de flujo y un menor costo computacional.
\end{abstract}

Palabras clave: Modelo de turbulencia, volúmenes finitos, sistema de distribución de aire acondicionado.

\begin{abstract}
In this investigation the finite volumes method was used to numerically simulate the thermofluiddynamic behavior of air in an air conditioning distribution system. The mathematical model that-governs the behavior of airflow in the distribution duct was described by means of applying a turbulence or closure model. For this purpose $k-\varepsilon, R N G$ - $k$ and Reynolds Tensions models were used individually. Three cases were simulated and the results obtained from these simulations indicate that the $k-\varepsilon$ model shows a better numerical behavior in the simulated problem, generating smaller residues in the flow variables and a reduced computing cost.
\end{abstract}

Keywords: Turbulence model, finite volumes, air conditioning distribution system.

\section{INTRODUCCIÓN}

Los flujos turbulentos están caracterizados por la presencia de campos de velocidad fluctuantes. Esas fluctuaciones mezclan las cantidades de transporte de momento, energía y concentración de especies, y causan que las cantidades transportadas también fluctúen. Como esas fluctuaciones pueden ser de pequeña escala y alta frecuencia, su simulación directa es muy costosa en cálculos prácticos de ingeniería. Para resolver este inconveniente las ecuaciones gobernantes instantáneas (exactas) pueden ser promediadas en el tiempo y en el espacio, obteniendo como resultado un conjunto de ecuaciones modificadas que son computacionalmente menos costosas de resolver. Estas ecuaciones modificadas contienen variables adicionales, las cuales deben ser resueltas en términos de cantidades conocidas mediante la aplicación de modelos de turbulencia.

La selección del modelo de turbulencia en un determinado caso dependerá de consideraciones como la física abarcada en el flujo, la práctica establecida para un tipo de problema en específico, el nivel de exactitud requerido, los recursos

\footnotetext{
1 Centro de Termofluidodinámica y Mantenimiento (CTYM). Universidad de Oriente. Puerto La Cruz, Anzoátegui, Apdo. 4547. Fax: 0281-2623270. Venezuela. E-mail: mariacolladoc@ cantv.net

2 Centro de Termofluidodinámica y Mantenimiento (CTYM). Universidad de Oriente. Puerto La Cruz, Anzoátegui, Apdo. 4547. Fax: 0281-2623270. Venezuela. E-mail: rmedgar5@cantv.net
} 
computacionales adecuados y la cantidad de tiempo disponible para la simulación.

En un sistema de distribución de aire acondicionado el flujo se caracteriza por presentar un comportamiento turbulento. Actualmente están disponibles una gran variedad de modelos de turbulencia y resulta de interés conocer cuál de ellos presenta un mejor comportamiento numérico en la simulación de este tipo de sistemas.

Esta investigación se realiza con el propósito de encontrar el modelo de turbulencia que resulte más eficiente en la simulación de sistemas de distribución de aire acondicionado. Para ello se realizan simulaciones numéricas del flujo en un sistema de distribución de aire acondicionado empleando el método de los volúmenes finitos mediante el paquete computacional $\mathrm{CFX}^{\mathrm{TM}}$, aplicando los modelos de turbulencia k- $\varepsilon$, RNG k- $\varepsilon$ y el modelo de las tensiones de Reynolds para una misma geometría del conducto y un mallado no estructurado.

Las comparaciones entre los casos simulados se establecerán en base a los residuos calculados para cada solución y al costo computacional de las mismas.

Estudios recientes han demostrado que el modelo de turbulencia empleado en un determinado problema tiene mucha influencia en la exactitud de los resultados obtenidos. En 1995, Rokni y Sunden [5] realizaron una investigación numérica sobre el modelado de la turbulencia de flujos en ductos cuadrados con convección forzada empleando el método de los volúmenes finitos. Ellos aplicaron cuatro modelos de turbulencia: el modelo k- $\varepsilon$ estándar, el modelo $\mathrm{k}-\varepsilon$ no lineal, el modelo algebraico de las tensiones de Reynolds y el modelo de las tensiones de Reynolds. En este trabajo los autores discutieron las ventajas y desventajas de cada modelo de turbulencia empleado, y las comparaciones fueron realizadas en función del factor de fricción y del número de Nusselt obtenido en cada caso. En el año 1997, Chen y Chao [8] emplearon la dinámica de fluidos computacional para predecir los detalles del flujo de aire en ambientes interiores con sistemas de ventilación de desplazamiento, con el objeto de encontrar el modelo de turbulencia que mejor se adaptara al caso de estudio. Para ello emplearon tres modelos de turbulencia, dos modelos de viscosidad de remolino (el modelo k- $\varepsilon$ estándar y el modelo RNG k- $\varepsilon$ ) y el modelo de las tensiones de Reynolds. En esta investigación los autores encontraron que los resultados obtenidos con los modelos de viscosidad de remolino no coincidieron con la data experimental, mientras que el modelo de las tensiones de Reynolds predijo satisfactoriamente las distribuciones de velocidad, temperatura y las cantidades turbulentas. En el año 1998, Chen y Xu [9] propusieron un nuevo modelo llamado Ecuación Cero para simular la distribución tridimensional de velocidad, temperatura y concentración de contaminantes en el flujo de aire en ambientes interiores. En su investigación los autores emplearon el nuevo modelo para predecir los diferentes tipos de convección: natural, forzada, mixta, así como la ventilación por desplazamiento en una habitación. Los resultados obtenidos se aproximaron a los datos experimentales y a los resultados arrojados por el modelo k- $\varepsilon$ estándar, con la ventaja de que el nuevo modelo es mucho más económico computacionalmente. En el año 2000, Valencia y Orellana [1] realizaron una simulación del flujo turbulento y la transferencia de calor alrededor de un cuerpo tridimensional de sección cuadrada utilizando el modelo $\mathrm{k}-\varepsilon$ estándar de turbulencia y una versión modificada, estableciendo comparaciones entre los resultados obtenidos para cada modelo. Ellos encontraron que con la versión modificada se obtuvieron mejores predicciones del coeficiente global de transferencia de calor de los cuerpos que con el modelo estándar.

\section{METODOLOGÍA}

\section{Modelo físico}

El modelo físico consiste en un sistema de distribución de aire acondicionado, diseñado mediante la aplicación del método de fricción constante, que recibe $0,57 \mathrm{~m}^{3} / \mathrm{s}$ de aire frío del equipo acondicionador y lo suministra al espacio mediante tres salidas de 8"x 8". Las características geométricas y dimensionales del sistema se muestran en las figuras 1 y 2 .

La figura 3 muestra la composición de las láminas de poliuretano que conforman el ducto de distribución y en las tablas 1 y 2 pueden verse las dimensiones de las láminas y sus propiedades, respectivamente.

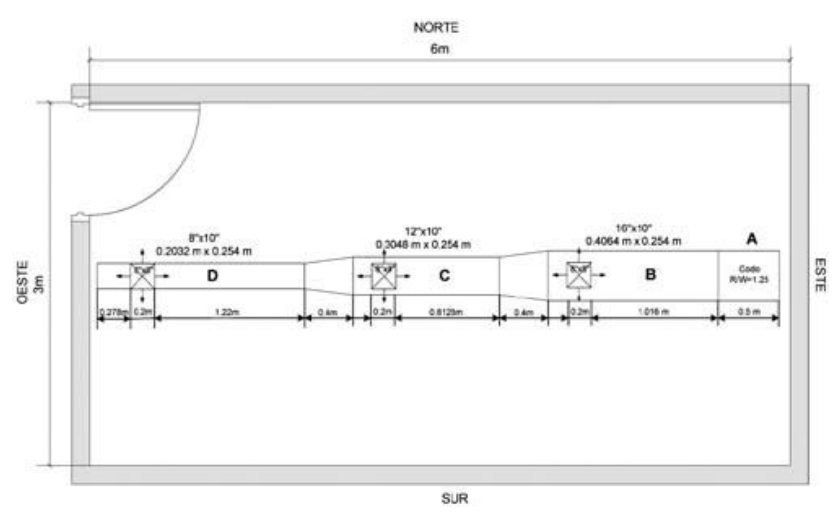

Figura 1. Dimensiones del sistema de distribución. 


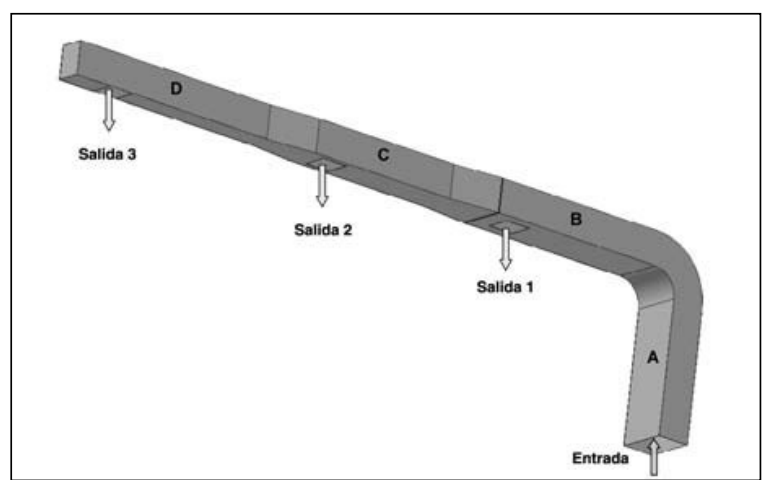

Figura 2. Vista isométrica del sistema de distribución.

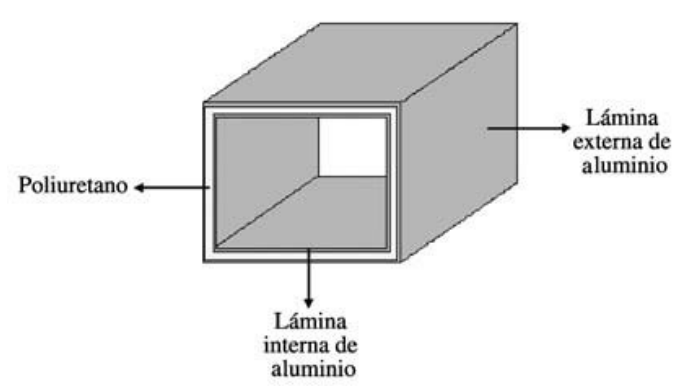

Figura 3. Composición de los paneles de poliuretano.

Tabla 1. Dimensiones de los paneles de poliuretano.

\begin{tabular}{|c|c|}
\hline Espesor láminas de aluminio & $8 \times 10^{-5} \mathrm{~m}$ \\
\hline Espesor poliuretano & $2 \times 10^{-2} \mathrm{~m}$ \\
\hline Espesor total del ducto & $0,02008 \mathrm{~m}$ \\
\hline
\end{tabular}

Tabla 2. Propiedades físicas y térmicas del poliuretano con recubrimiento de aluminio.

\begin{tabular}{|l|c|}
\hline Densidad & $48-50 \mathrm{~kg} / \mathrm{m}^{3}$ \\
\hline Presión interna máxima & $500 \mathrm{~Pa}$ \\
\hline Rugosidad absoluta & $0,00012 \mathrm{~m}$ \\
\hline Conductividad térmica a $283 \mathrm{~K}$ & $0,022 \mathrm{~W} / \mathrm{m} . \mathrm{K}$ \\
\hline
\end{tabular}

\section{Modelo matemático}

El modelo matemático de este sistema de distribución está conformado por la ecuación de continuidad, las ecuaciones de movimiento y la ecuación de conservación de la energía, aplicadas a un flujo tridimensional, estacionario y no isotérmico de un fluido viscoso con propiedades físicas constantes. Este modelo matemático emplea varias simplificaciones que facilitan su solución pero sin alterar su validez [6]; estas simplificaciones se presentan a continuación:

a) Flujo incompresible

b) Fluido viscoso newtoniano c) Fluido con propiedades físicas constantes: $\rho, \mu$ y K

d) Estado estacionario.

Como resultado de las idealizaciones citadas anteriormente la ecuación de continuidad, y de la aplicación del concepto de la media temporal de Reynolds, las ecuaciones de movimiento y la ecuación de la energía, expresadas en coordenadas rectangulares, quedan simplificadas de la siguiente manera:

Ecuación de continuidad de tiempo ajustado en su forma vectorial

$$
(\nabla \cdot \bar{v})=0
$$

Ecuación de movimiento de tiempo ajustado en su forma vectorial

$$
\rho \nabla \bullet(\bar{v} \otimes \bar{v})=-\nabla \bar{p}-\left[\nabla \bullet \bar{\tau}^{(l)}\right]-\left[\nabla \bullet \bar{\tau}^{(t)}\right]+\rho g_{z}
$$

donde:

$$
\bar{\tau}_{x x}{ }^{(t)}=\rho \overline{v_{x}{ }^{\prime} v_{x}{ }^{\prime}} ; \quad \bar{\tau}_{x y}{ }^{(t)}=\rho \overline{v_{x}{ }^{\prime} v_{y}{ }^{\prime}} ; \quad \text { etc. }
$$

$$
\bar{\tau}_{x x}{ }^{(l)}=-\mu\left[2 \frac{\partial v_{x}}{\partial x}\right] ; \quad \bar{\tau}_{y y}{ }^{(l)}=-\mu\left[2 \frac{\partial v_{y}}{\partial y}\right] ; \text { etc. }
$$

Ecuación de la energía de tiempo ajustado en forma vectorial

$$
\begin{aligned}
& \rho \hat{C} v \nabla \bullet(\bar{T} \bar{v})=-\left[\nabla \bullet \bar{q}^{(l)}\right]-\left[\nabla \bullet \bar{q}^{(t)}\right] \\
&+\mu \bar{\Phi}_{v}{ }^{(l)}+\mu \bar{\Phi}_{v}{ }^{(t)}
\end{aligned}
$$

donde:

$$
\begin{gathered}
\bar{q}^{(l)}=-K \nabla^{2} \bar{T} \\
\bar{q}_{x}^{(t)}=\rho \hat{c}_{V} \overline{v_{x}{ }^{\prime} T^{\prime}} ; \quad \bar{q}_{y}^{(t)}=\rho \hat{c}_{V} \overline{v_{y}{ }^{\prime} T^{\prime}} \\
\bar{q}_{z}^{(t)}=\rho \hat{C}_{V} \overline{v_{z}{ }^{\prime} T^{\prime}} \\
\bar{\Phi}_{v}^{(l)}=2\left\{\left(\frac{\partial \bar{v}_{x}}{\partial x}\right)^{2}+\left(\frac{\partial \bar{v}_{y}}{\partial y}\right)^{2}+\left(\frac{\partial \bar{v}_{z}}{\partial z}\right)^{2}\right\}+ \\
\left\{\left(\frac{\partial \bar{v}_{x}}{\partial y}+\frac{\partial \bar{v}_{y}}{\partial x}\right)^{2}+\left(\frac{\partial \bar{v}_{x}}{\partial z}+\frac{\partial \bar{v}_{z}}{\partial x}\right)^{2}+\left(\frac{\partial \bar{v}_{y}}{\partial z}+\frac{\partial \bar{v}_{z}}{\partial y}\right)^{2}\right\}
\end{gathered}
$$




$$
\bar{\Phi}_{v}{ }^{(t)}=\sum_{i=1}^{3} \sum_{j=1}^{3}\left(\overline{\frac{\partial v_{i}{ }^{\prime}}{\partial x_{j}} \frac{\partial v_{i}{ }^{\prime}}{\partial x_{j}}}+\overline{\frac{\partial v_{i}{ }^{\prime}}{\partial x_{j}} \frac{\partial v_{j}{ }^{\prime}}{\partial x_{i}}}\right)
$$

\section{Modelos de turbulencia}

Los modelos de turbulencia son utilizados para predecir los efectos de la turbulencia en un flujo sin necesidad de resolver todas las pequeñas escalas de las fluctuaciones turbulentas. Han sido desarrollados una variedad de modelos basados en las ecuaciones de Navier Stokes promediadas por Reynolds (RANS) que pueden ser empleados para modelar la turbulencia [7]. Algunos de estos modelos tienen aplicaciones específicas, mientras que otros pueden ser aplicados en un amplio rango de flujos con un grado razonable de exactitud. Los modelos RANS pueden ser clasificados en modelos de viscosidad de remolino y en modelos de las tensiones de Reynolds [2].

En el presente trabajo se emplearán dos modelos de viscosidad remolino ( $k-\varepsilon$ y RNG k- $\varepsilon$ ) y el modelo de las tensiones de Reynolds para resolver las fluctuaciones turbulentas presentes en el flujo de aire del sistema de aire acondicionado. A continuación se presentan las formulaciones matemáticas que rigen cada uno de esos tres modelos.

\section{Modelo $k-\varepsilon$}

Uno de los modelos de turbulencia más prominentes, el $\mathrm{k}-\varepsilon$, ha sido implementado en la mayoría de los códigos de dinámica de fluidos computacional y es considerado el modelo estándar de la industria. Este modelo ha sido extensamente validado y ha demostrado ser estable y numéricamente robusto. En el software $\mathrm{CFX}^{\mathrm{TM}}$ el modelo de turbulencia k- $\varepsilon$ utiliza la aproximación de la función de pared para mejorar la robustez y precisión de los resultados cuando el mallado en la región cercana a la pared es muy fino.

La energía cinética turbulenta, $\mathrm{k}$, está definida como la variación de las fluctuaciones en la velocidad y tiene las dimensiones de $\left(\mathrm{m}^{2} / \mathrm{s}^{2}\right)$, $\varepsilon$ es la disipación de remolino turbulenta (la tasa a la cual las fluctuaciones de velocidad se disipan) y tiene las dimensiones de $\left(\mathrm{m}^{2} / \mathrm{s}^{3}\right)$.

El modelo k- $\varepsilon$ está basado en el concepto de la viscosidad remolino, y asume que la viscosidad turbulenta está ligada a la energía cinética turbulenta y la disipación mediante la relación:

$$
\mu_{t}=C_{\mu} \rho\left(\frac{k^{2}}{\varepsilon}\right)
$$

donde, $C_{\mu}$ es una constante. Los valores de $\mathrm{k}$ y $\varepsilon$ vienen directamente de las ecuaciones diferenciales de transporte de la energía cinética turbulenta y de la tasa de disipación turbulenta.

$$
\begin{gathered}
\rho \nabla \bullet(\bar{v} k)=\nabla \bullet\left[\left(\mu+\frac{\mu_{t}}{\sigma_{k}}\right) \nabla k\right]+P_{k}-\rho \varepsilon \\
\rho \nabla \bullet(\bar{v} \varepsilon)=\nabla \bullet\left[\left(\mu+\frac{\mu_{t}}{\sigma_{\varepsilon}}\right) \nabla \varepsilon\right] \\
+\frac{\varepsilon}{k}\left(C_{\varepsilon 1} P_{k}-C_{\varepsilon 2} \rho \varepsilon\right)
\end{gathered}
$$

donde, $\mathrm{C}_{\varepsilon 1}, \mathrm{C}_{\varepsilon 2}, \sigma_{\mathrm{k}}$ y $\sigma_{\varepsilon}$ son constantes, $\mathrm{P}_{\mathrm{k}}$ es la producción turbulenta debido a las fuerzas viscosas y a las fuerzas de flotación, la cual es modelada empleando la siguiente ecuación:

$$
P_{k}=\mu_{t} \nabla \bar{v} \bullet\left(\nabla \bar{v}+\nabla \bar{v}^{-T}\right)+P_{k b}
$$

Empleando el modelo de flotación de Boussinesq, $\mathrm{P}_{\mathrm{kb}}$ es el término de producción de la flotación y está definido de la siguiente manera:

$$
P_{k b}=\frac{\mu_{t}}{\operatorname{Pr}_{t}} \rho \beta g \bullet \nabla T
$$

\section{Modelo $R N G$ - $\varepsilon$}

Este modelo está desarrollado usando técnicas de renormalización de la teoría de grupo. Las ecuaciones de transporte para la generación y disipación de turbulencia son las mismas que las empleadas en el modelo k- $\varepsilon$ estándar, pero las constantes utilizadas son diferentes y la constante $\mathrm{C}_{\varepsilon 1}$ es remplazada por la función $\mathrm{C}_{\varepsilon 1 \mathrm{RNG}}$.

La ecuación de disipación turbulenta queda entonces expresada de la siguiente forma:

$$
\begin{gathered}
\rho \nabla \bullet(\bar{v} \varepsilon)=\nabla \bullet\left[\left(\mu+\frac{\mu_{t}}{\sigma_{\varepsilon R N G}}\right) \nabla \varepsilon\right] \\
+\frac{\varepsilon}{k}\left(C_{\varepsilon 1 R N G} P_{k}-C_{\varepsilon 2 R N G} \rho \varepsilon\right)
\end{gathered}
$$

donde:

$$
C_{\varepsilon 1 R N G}=1,42-f_{\eta}
$$




$$
\begin{gathered}
f_{\eta}=\frac{\eta\left(1-\frac{\eta}{4,38}\right)}{\left(1+\beta_{R N G} \eta^{3}\right)} \\
\eta=\sqrt{\frac{P_{k}}{\rho C_{\mu R N G} \varepsilon}}
\end{gathered}
$$

Modelo estándar de las tensiones de Reynolds

Este modelo está basado en la ecuación para la disipación de remolino turbulenta $\varepsilon$ y emplea las siguientes ecuaciones de transporte para los esfuerzos de Reynolds:

$$
\begin{gathered}
\nabla \bullet\left(\rho \bar{v} \otimes \overline{v^{\prime} \otimes v^{\prime}}\right)=P+\phi \\
+\nabla \cdot\left(\left(\mu+\frac{2}{3} C_{S} \rho \frac{k^{2}}{\varepsilon}\right) \overline{\nabla v^{\prime} \otimes v^{\prime}}\right)-\frac{2}{3} \rho \varepsilon \delta
\end{gathered}
$$

donde $\mathrm{C}_{\mathrm{S}}$ es una constante del modelo, $\phi$ es la correlación esfuerzo-presión y $\mathrm{P}$ es el término exacto de producción, dado por:

$$
P=-\rho\left(\overline{v^{\prime} \otimes v^{\prime}}(\nabla \bar{v})^{T}+(\nabla \bar{v}) \overline{v^{\prime} \otimes v^{\prime}}\right)
$$

Como la disipación turbulenta aparece en las ecuaciones de esfuerzos individuales todavía es requerida una ecuación para $\varepsilon$. Esta ecuación tiene ahora la forma:

$$
\begin{aligned}
& \rho \nabla \bullet(\bar{v} \varepsilon)=\frac{\varepsilon}{k}\left(C_{\varepsilon 1} P-C_{\varepsilon 2} \rho \varepsilon\right) \\
& +\nabla \bullet\left[\left(\mu+\frac{\mu_{t}}{\sigma_{\varepsilon}}\right) \nabla \varepsilon\right]
\end{aligned}
$$

donde $\mathrm{C}_{\varepsilon 1}, \mathrm{C}_{\varepsilon 2}$ son constantes cuyos valores se muestran en la tabla 3 .

Tabla 3. Constantes empleadas en el modelo estándar de las tensiones de Reynolds.

\begin{tabular}{|c|l|l|l|}
\hline Constante & Valor & Constante & Valor \\
\hline $\mathrm{C}_{\mu \mathrm{RS}}$ & 1,7 & $\mathrm{C}_{\mathrm{S} 2}$ & $-1,05$ \\
\hline $\mathrm{S}_{\mathrm{eRS}}$ & 1,36 & $\mathrm{C}_{\mathrm{r} 1}$ & 0,9 \\
\hline $\mathrm{C}_{\mathrm{s}}$ & 0,22 & $\mathrm{C}_{\mathrm{r} 2}$ & 0,8 \\
\hline $\mathrm{C}_{\varepsilon 1}$ & 1,45 & $\mathrm{C}_{\mathrm{r} 3}$ & 0,65 \\
\hline $\mathrm{C}_{\mathrm{e} 2}$ & 1,83 & $\mathrm{C}_{\mathrm{r} 4}$ & 0,625 \\
\hline $\mathrm{C}_{\mathrm{S} 1}$ & 1,7 & $\mathrm{C}_{\mathrm{r} 5}$ & 0,2 \\
\hline
\end{tabular}

Uno de los términos más importantes en el modelo de las tensiones de Reynolds es la correlación esfuerzo-presión, $\phi_{i j}$, la cual puede ser expresada de forma general como sigue:

$$
\phi_{i j}=\phi_{i j 1}+\phi_{i j 2}
$$

donde:

$$
\begin{gathered}
\phi_{i j 1}=-\rho \varepsilon\left(C_{s 1} a+C_{s 2}\left(a a-\frac{1}{3} a \bullet a \delta\right)\right) \\
\phi_{i j 2}=-C_{r 1} P . a+C_{r 2} \rho k S-C_{r 3} \rho k S \sqrt{a \bullet a} \\
+C_{r 4} \rho k\left(a S^{T}+S a^{T}-\frac{2}{3} a \bullet S \delta\right) \\
+C_{r 5} \rho k\left(a W^{T}+W a^{T}\right) \\
a=\frac{\overline{v^{\prime} \otimes v^{\prime}}}{k}-\frac{2}{3} \delta \\
S=\frac{1}{2}\left(\nabla \bar{v}+(\nabla \bar{v})^{T}\right) \\
W=\frac{1}{2}\left(\nabla \bar{v}-(\nabla \bar{v})^{T}\right)
\end{gathered}
$$

En esta formulación, a es el tensor anisotrópico, S es la tasa de deformación y $\mathrm{W}$ es la vorticidad.

\section{Condiciones de contorno}

Una vez planteadas todas las ecuaciones de gobierno que rigen el comportamiento del flujo de aire en el sistema de distribución es necesario definir las condiciones de contorno que permitirán dar solución a esas ecuaciones de conservación. Las características geométricas del dominio en estudio permitieron hacer las simulaciones considerando sólo la mitad de la geometría. Esto es una simplificación que se hace con el propósito de disminuir el costo computacional de la simulación aunque se sabe que es posible que esta aproximación introduzca cierto porcentaje de error en los resultados de la simulación.

Las condiciones de borde del problema fueron impuestas en el dominio de la siguiente manera: 
- Entrada:

$$
\begin{gathered}
v_{x}=0 \quad p=p_{e} \\
v_{y}=0 \quad k=k_{e} \\
v_{z}=v_{e} \quad \varepsilon=\varepsilon_{e} \\
T=T_{e}
\end{gathered}
$$

donde el subíndice $e$ indica los valores de entrada de las variables.

- Salidas:

$$
\begin{aligned}
p & =p_{s} \\
k & =k_{s} \\
\varepsilon & =\varepsilon_{s}
\end{aligned}
$$

donde el subíndice $s$ indica los valores de salida de las variables.

- Paredes:

Condición de no deslizamiento:

$$
V_{\text {fluido }}=V_{\text {pared }}=0
$$

Coeficiente de transferencia de calor:

$$
q_{w}=U\left(T_{b}-T_{n w}\right)
$$

Donde U es el coeficiente global de transferencia de calor especificado, $\mathrm{T}_{\mathrm{b}}$ es la temperatura de contorno especificada $\mathrm{y}_{\mathrm{nw}}$ es la temperatura en el nodo próximo a la pared.

\section{Solución numérica}

Las ecuaciones diferenciales con sus respectivas condiciones de borde descritas en el modelo matemático fueron resueltas a través del código comercial de Dinámica de Fluidos Computacional $\mathrm{CFX}^{\mathrm{TM}}$, para el cual tiene la solución basado en el método de los volúmenes finitos para mallas no estructuradas.

Para la construcción del mallado no estructurado se empleó el método de Avance Frontal e Inflación, el cual produce una malla de superficie triangular empleando el método de Delaunay y una malla de volumen, mediante el método de Avance Frontal, que puede contener elementos en forma de tetraedros, prismas y pirámides [3, 4, 10]. En la figura 4 se muestra la malla generada por este método.

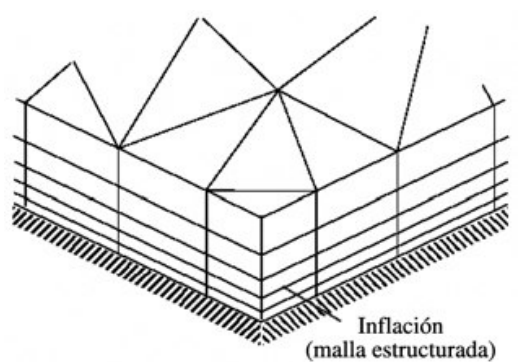

Figura 4. Mallado empleando el mecanismo de inflación.

En el dominio en estudio se construyó una malla no estructurada con refinamientos locales en las zonas próximas a las salidas de aire, se empleó el mecanismo de inflación en todas las paredes de la geometría y además se construyó una malla más refinada en la zona final del ducto para observar con mayor exactitud el fenómeno de convección libre existente en esa región del dominio.

En las figuras 5 y 6 se presenta un corte transversal y longitudinal de la malla construida.

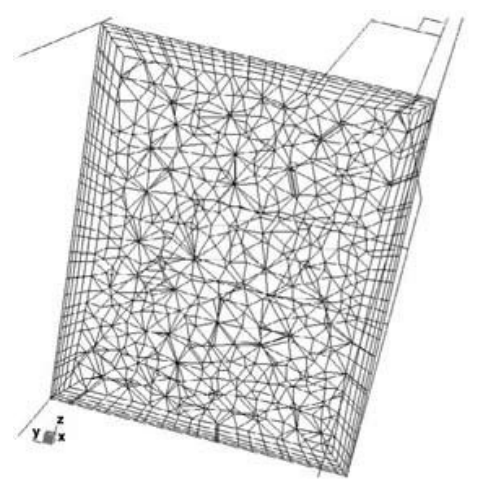

Figura 5. Corte transversal de la malla no estructurada.

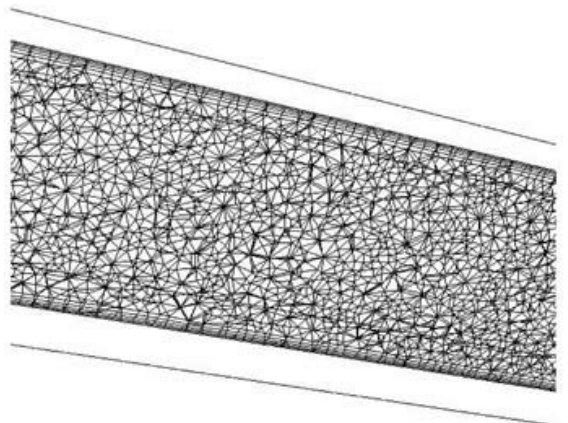

Figura 6. Corte longitudinal (izquierdo) de la malla no estructurada. 
En la figura 7 se muestra el refinamiento de la malla en las zonas cercanas a las salidas de aire, este refinamiento se logró gracias al uso de un controlador de malla ubicado en la línea central de las salidas de aire. En la figura 8 se muestra el refinamiento de la malla en la zona final del conducto.

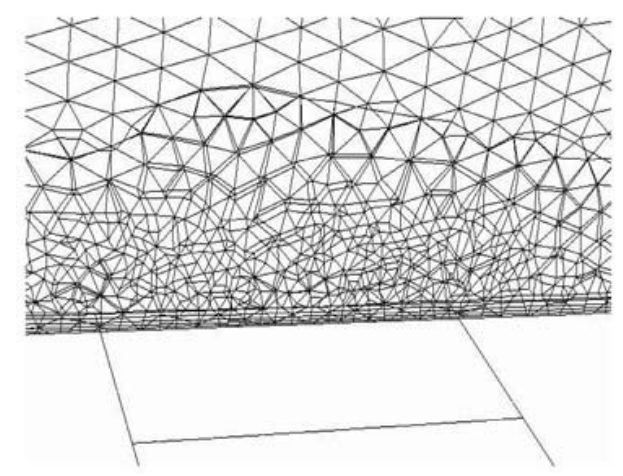

Figura 7. Refinamiento de la malla en la zona cercana a las salidas de aire.

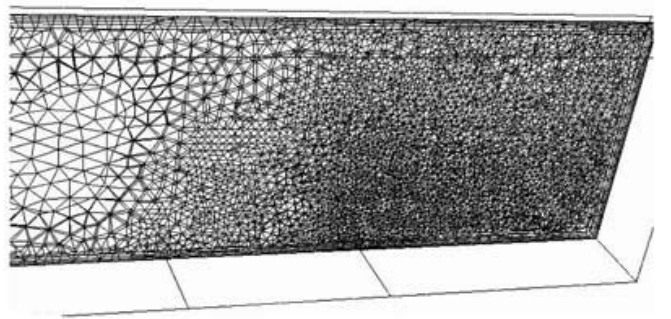

Figura 8. Refinamiento de la malla en la zona final del ducto.

\section{Parámetros de solución}

Para cada caso simulado se emplearon los parámetros de solución adecuados con el objeto de obtener, en la medida de lo posible, una buena tasa de convergencia. Variados algunos de ellos. A continuación se explican de forma general las características de cada parámetro de solución.

\section{Acoplamiento presión-velocidad}

El software CFX ${ }^{\mathrm{TM}}$ utiliza un solver acoplado, el cual resuelve las ecuaciones hidrodinámicas (para la presión y la velocidad) como un sistema individual. Esta técnica de solución utiliza una discretización implícita completa de las ecuaciones en cualquier paso de tiempo.

Este software utiliza una malla colocada "unstaggered" para resolver el acoplamiento presión velocidad mediante la adición de un término adicional a la ecuación de conservación de la masa.

\section{Técnica multimalla algebraica (AMG)}

El proceso de esta técnica comprende la solución de las ecuaciones de conservación para una malla fina en las primeras iteraciones y luego en mallas "virtuales" progresivamente más gruesas. Los resultados obtenidos para la malla más gruesa son entonces interpolados hacia la malla fina original.

\section{Esquema de advección}

Esquema de alta resolución: en este esquema el factor de mezcla varía a través del dominio basado en el campo de solución local. En regiones de flujo con gradientes de variables bajos, el factor de mezcla será cercano a uno. En áreas donde los gradientes cambien bruscamente el factor de mezcla será cercano a cero. Este esquema fue empleado en las simulaciones con el modelo $\mathrm{k}-\varepsilon$ con los dos tipos de discretización espacial y para los dos materiales.

\section{Inicialización}

En los dos casos simulados en este trabajo fueron empleados valores iniciales automáticos para las diferentes variables del flujo.

\section{Criterio de convergencia}

Para juzgar la convergencia del problema en estudio se seleccionó el límite de residual como $1 \times 10^{-5}$ en norma RMS.

\section{RESULTADOS}

A continuación se muestran los resultados obtenidos para los tres casos de estudio, empleando para todas las simulaciones un ordenador con las siguientes características:

- $\quad$ Procesador Intel Pentium IV de $2.8 \mathrm{GHz}$

- 1 GB de Memoria RAM

- $\quad$ Tarjeta de Video Ge Force FX Serie 5200 de 256 MB

- Disco Duro SATA de 80 GB

\section{Modelo de turbulencia k- $\varepsilon$}

Esta simulación requirió 261 iteraciones con un tiempo de cálculo de 8 horas con 16 minutos para alcanzar los niveles de convergencia requeridos, obteniéndose un 
rango de variación de temperatura en el dominio de $3,3648 \mathrm{~K}$.

La figura 9 muestra las curvas de residual en norma RMS para las velocidades y la masa obtenidas en este caso simulado. Este gráfico muestra que la velocidad U es la variable que requirió mayor número de iteraciones para alcanzar el nivel de convergencia especificado; esto se debe a que es la componente del vector velocidad que sufre mayores fluctuaciones a lo largo del dominio por ser la velocidad principal del flujo. Adicionalmente se observa que la curva de residual de masa es la que alcanza el nivel de convergencia requerido en menor número de iteraciones y su residuo final es del orden de $3,3 \times 10^{-7}$ RMS, mucho menor que el residuo final del resto de las variables, comportamiento que indica que la acumulación de masa en el dominio es bastante pequeña.

Se observa que en las curvas de las velocidades U y $\mathrm{W}$ se presentan oscilaciones repetitivas que dificultan la convergencia de la solución; esto puede deberse a distorsiones en la malla o a errores provenientes de la discretización de los términos convectivos en las ecuaciones de gobierno.

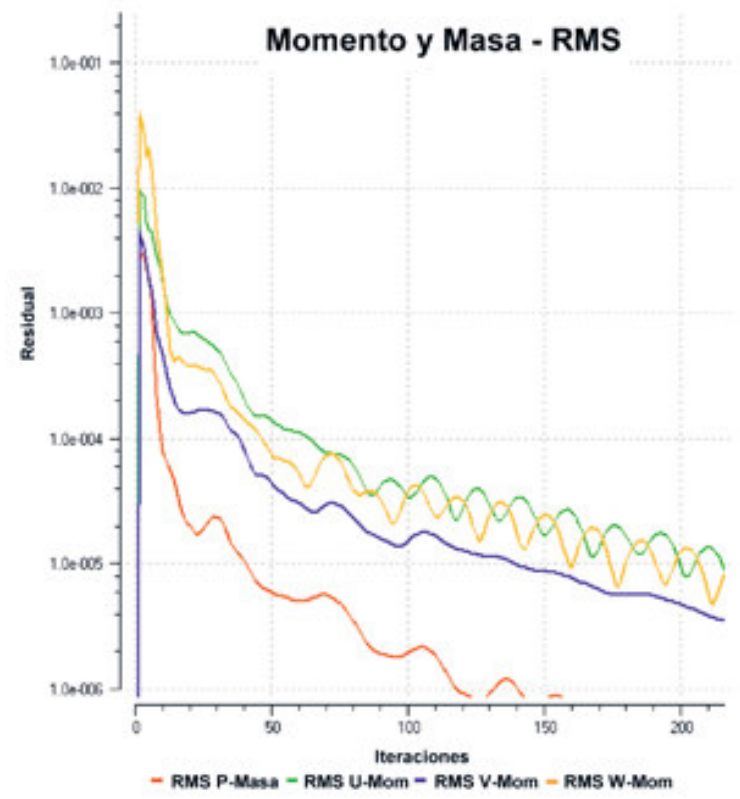

Figura 9. Curvas de residual en norma RMS para las ecuaciones de masa y momento en la simulación con el modelo de turbulencia k- $\varepsilon$.

La curva de residual en norma RMS para la entalpía se muestra en la figura 10. En este gráfico es posible notar que la entalpía alcanza el nivel de convergencia requerido mucho antes que las velocidades, en la iteración 70 aproximadamente, y su residuo final es también más pequeño.

Las curvas de residual en norma RMS para la energía cinética turbulenta $\mathrm{k}$ y su tasa de disipación $\varepsilon$ se muestran en la figura 11. El software $\mathrm{CFX}^{\mathrm{TM}}$ no toma en cuenta estas variables al momento de aplicar el criterio de convergencia especificado por el usuario; a pesar de esto se observa que tanto $\mathrm{k}$ como $\varepsilon$ alcanzan el límite de residual de $1 \times 10^{-5}$ RMS.

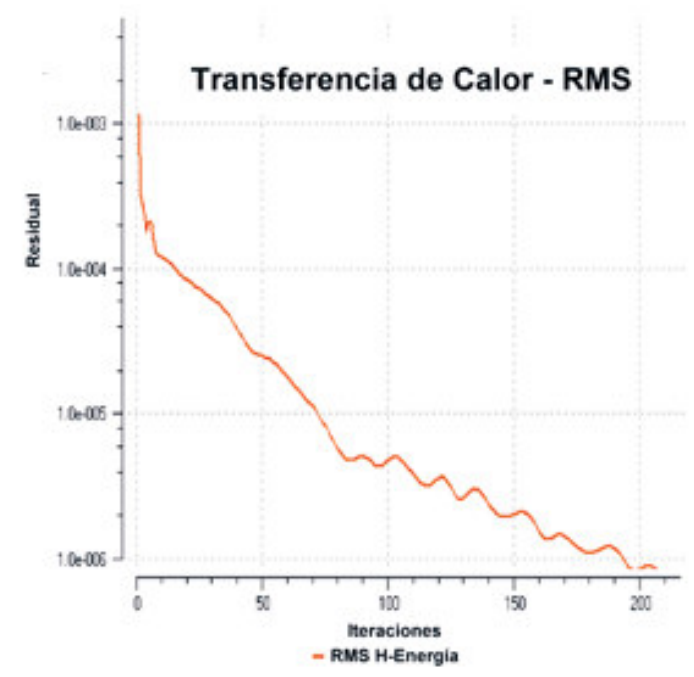

Figura 10. Curva de residual en norma RMS para la ecuación de la energía en la simulación con el modelo de turbulencia k- $\varepsilon$.

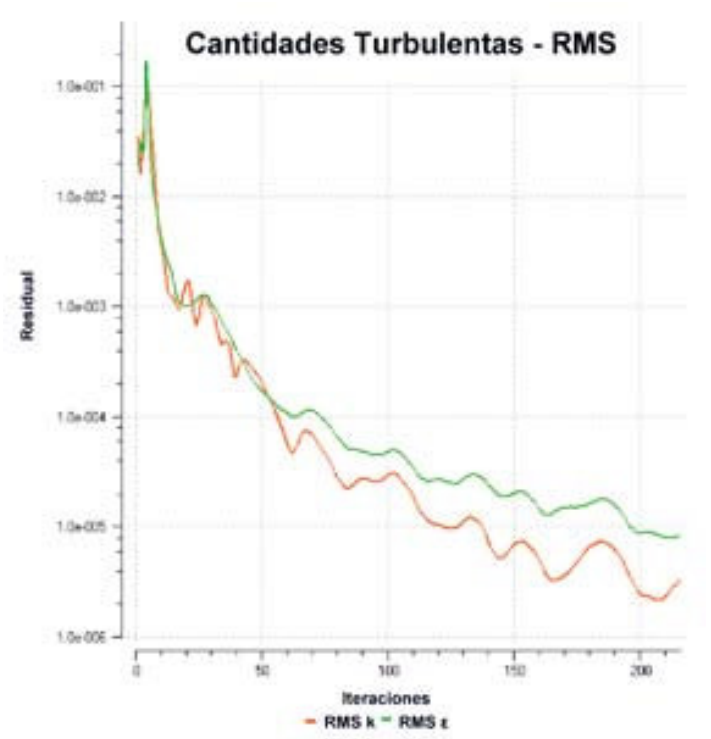

Figura 11. Curva de residual en norma RMS para la energía cinética turbulenta y su tasa de disipación en la simulación con el modelo de turbulencia k- $\varepsilon$. 


\section{Modelo de turbulencia RNG k- $\varepsilon$}

En esta simulación fue imposible alcanzar el nivel de convergencia de $1 \times 10^{-5}$ RMS debido a que se presentaron grandes oscilaciones en los residuales de las variables. Esas oscilaciones predecían que si los residuos de las variables de flujo disminuían hasta el límite de convergencia sería en un tiempo de cálculo muy elevado. Por esta razón se decidió aumentar el valor del residual a $1 \times 10^{-4}$ RMS para disminuir el costo de la simulación.

Este proceso de solución concluyó a las 327 iteraciones con un tiempo de cálculo de 11 horas y 34 minutos, obteniéndose un rango de variación de temperatura en el dominio de 3,2255 K.

En la figura 12 se muestran las curvas de residual en norma RMS para las velocidades y la masa obtenidas en este caso simulado. Al comparar estas curvas con las obtenidas para el caso anterior se observa que con el modelo k- $\varepsilon$ el valor del residual $1 \times 10^{-4}$ es alcanzado para todas las variables en menos iteraciones y con menos oscilaciones que con el modelo RNG k- $\varepsilon$.

La curva de residual en norma RMS para la entalpía se muestra en la figura 13. En este gráfico se observa que, a pesar de los problemas obtenidos para alcanzar la convergencia en las velocidades, la entalpía logró alcanzar un valor de $3 \times 10^{-5}$ RMS y en muchas menos iteraciones que las requeridas para que las velocidades alcanzaran el límite de $1 \times 10^{-4}$ RMS.

Las curvas de residual en norma RMS para la energía cinética turbulenta $\mathrm{k}$ y su tasa de disipación $\varepsilon$ obtenidas para este caso se muestran en la figura 14. Estas curvas presentan un comportamiento similar a las del modelo k- $\varepsilon$ con la diferencia de que sus residuos son más elevados.

\section{Modelo de turbulencia de las tensiones de Reynolds (RSM)}

Debido al alto costo computacional del modelo de turbulencia de las tensiones de Reynolds, en este caso fue necesario elevar el límite convergencia a $1 \times 10^{-4}$. A pesar de esto el tiempo de cálculo obtenido para esta simulación fue de 21 horas y 53 minutos, tiempo mucho mayor al requerido con los dos modelos anteriores. El número de iteraciones realizadas fue 418 , obteniéndose un rango de variación de la temperatura en el dominio de $3,5250 \mathrm{~K}$.

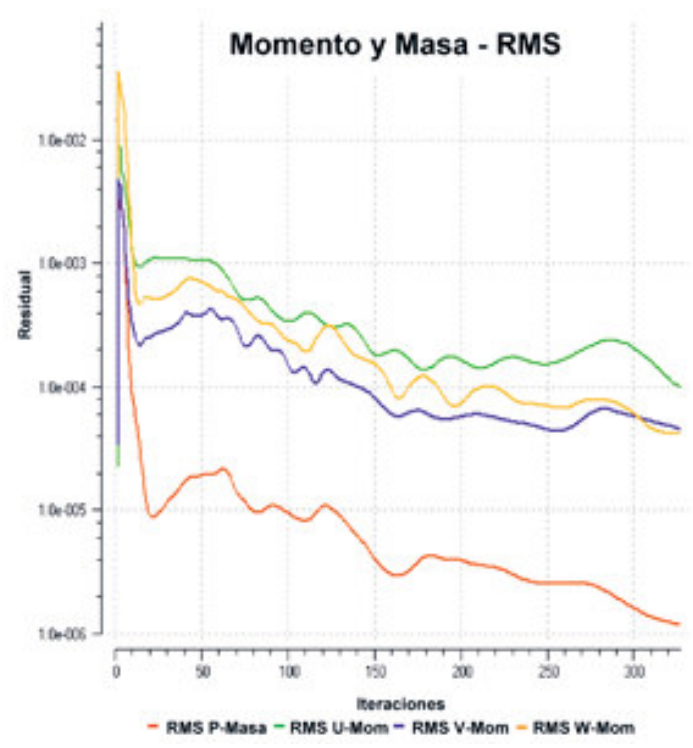

Figura 12. Curvas de residual en norma RMS para las ecuaciones de masa y momento en la simulación con el modelo de turbulencia RNG k- $\varepsilon$.

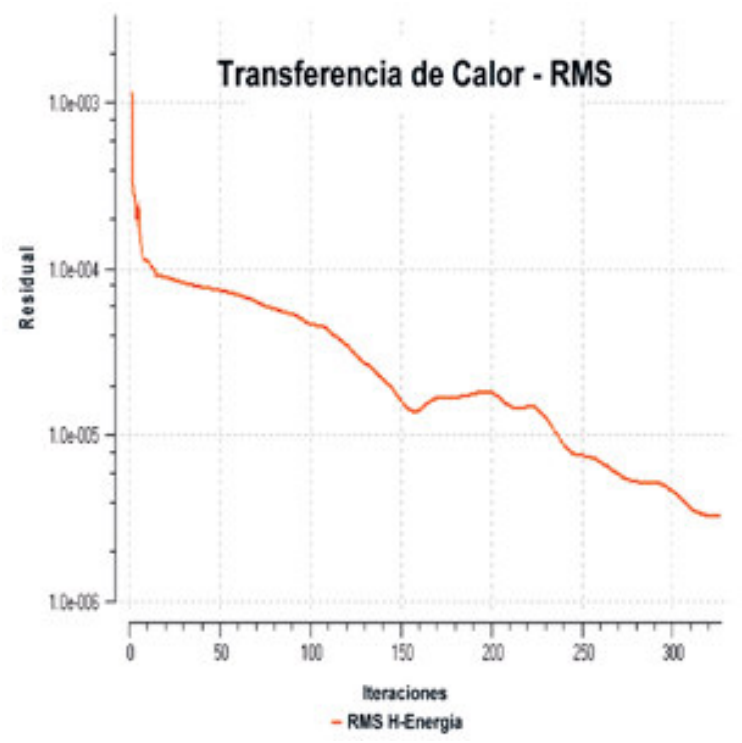

Figura 13. Curva de residual en norma RMS para la ecuación de la energía en la simulación con el modelo de turbulencia RNG k- $\varepsilon$.

En las figuras 15 y 16 se muestran las curvas de residual de las velocidades, masa y de la entalpía respectivamente. Estas curvas presentan oscilaciones marcadas con un comportamiento similar al presentado en el modelo RNG $\mathrm{k}-\varepsilon$, con la diferencia de que la velocidad $\mathrm{U}$, la cual como en los casos anteriores presenta los mayores residuos, alcanza el límite de convergencia a las 400 iteraciones con un tiempo de cálculo mucho mayor que para los dos 
casos anteriores. Para la entalpía el residual alcanzado fue de $3 \times 10^{-5}$ aproximadamente, valor muy similar al obtenido en el caso del modelo RNG k-E.

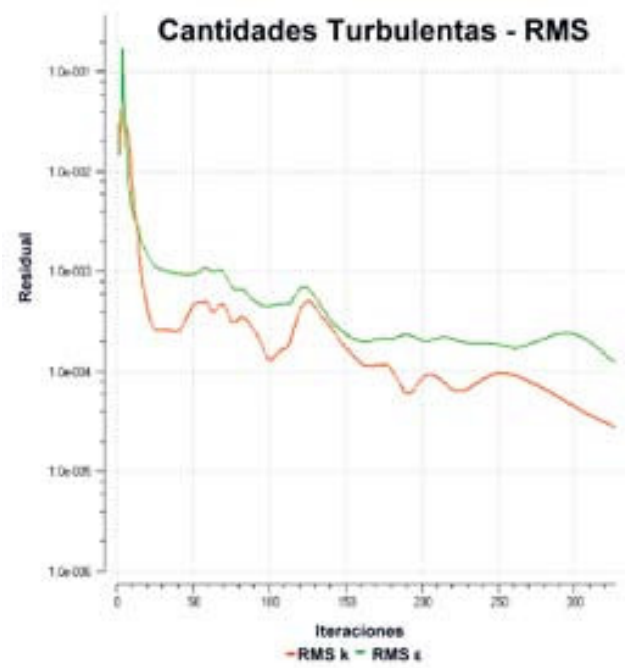

Figura 14. Curvas de residual en norma RMS para la energía cinética turbulenta y su tasa de disipación en la simulación con el modelo de turbulencia RNG k- $\varepsilon$.

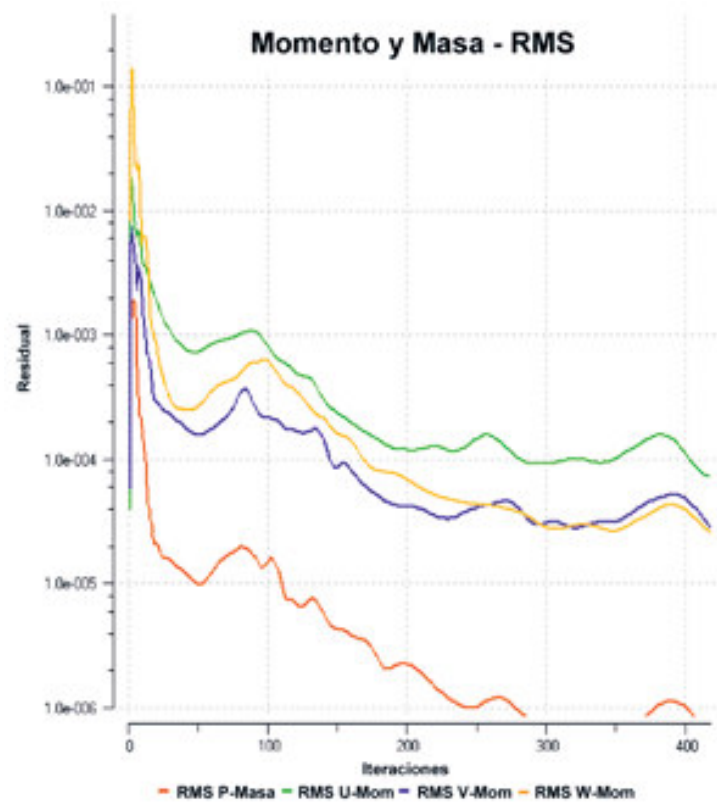

Figura 15. Curva de residual en norma RMS para las ecuaciones de masa y momento en la simulación con malla no estructurada y modelo RSM.

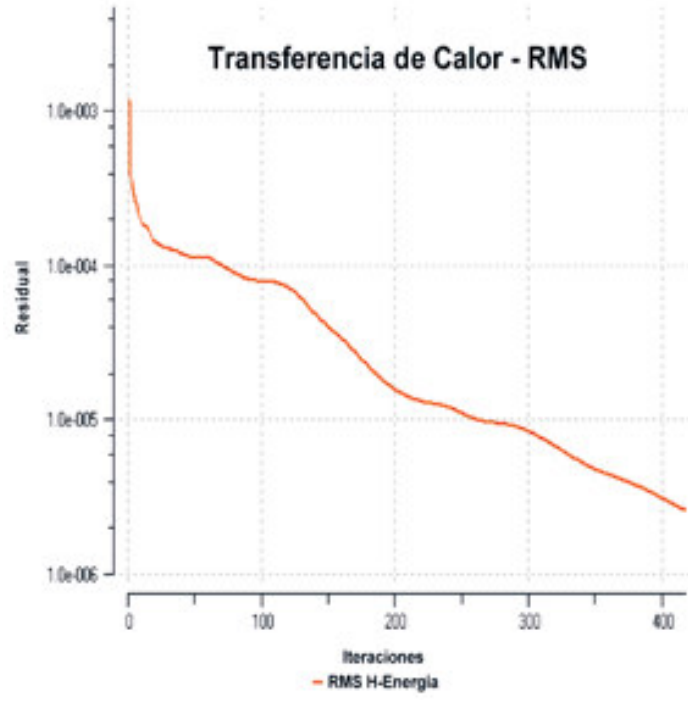

Figura 16. Curva de residual en norma RMS para la ecuación de la energía en la simulación con malla no estructurada y modelo RSM.

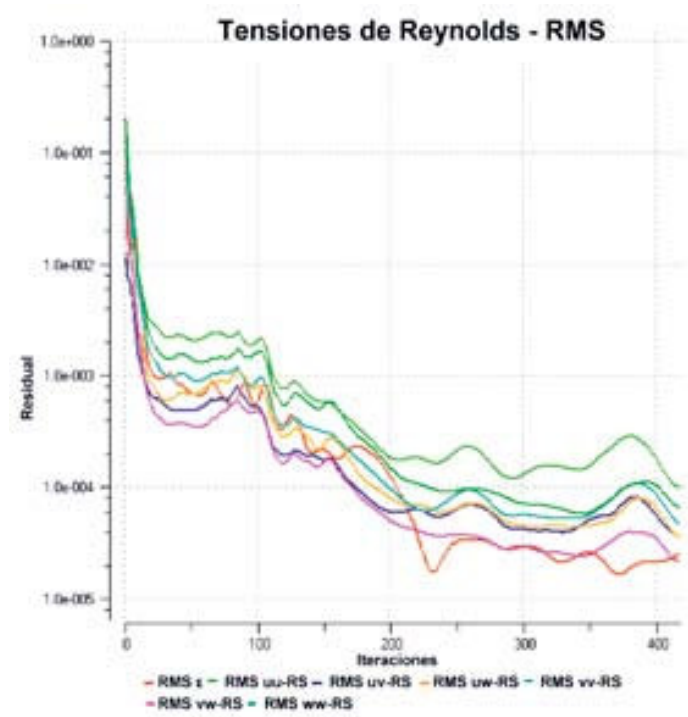

Figura 17. Curvas de residual en norma RMS para las tensiones de Reynolds en la simulación con malla no estructurada y modelo RSM.

Por su parte, las tensiones de Reynolds (figura 17), variables características de este modelo, son las que requirieron mayor número de iteraciones para lograr la convergencia, y entre ellas la que presenta mayores residuos es la componente uu-RS, la cual corresponde al promedio del producto de la velocidad fluctuante en la dirección del flujo. 


\section{Comparación de los tiempos de cálculo de las simulaciones}

En la sección anterior se observó cómo el comportamiento de la convergencia varía para los tres casos simulados. Ahora es necesario realizar comparaciones entre los tiempos de cálculo de las simulaciones y los residuos finales de cada variable con el objeto de seleccionar adecuadamente el caso que mejor se adapte al problema simulado.

En la figura 18 se muestra un gráfico de barras en el cual se comparan los tiempos de cálculo consumidos por cada caso simulado, resultando evidente el ahorro computacional que representa emplear el modelo k- $\varepsilon$ en la simulación de este sistema de distribución.

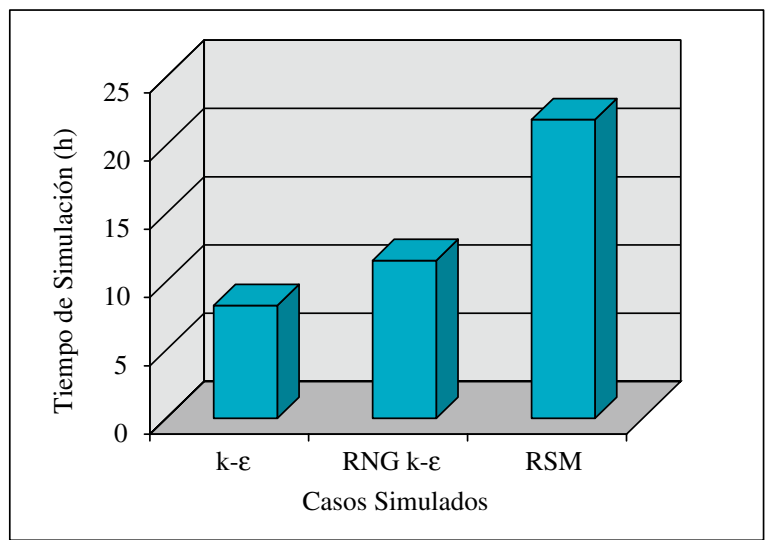

Figura 18. Comparación entre los tiempos de cálculo para los casos simulados.

Mediante la figura 19 es posible hacer comparaciones entre los residuos finales obtenidos para las variables del flujo. En esta figura se observa que para los tres casos simulados la variable que presenta menores residuos es la masa seguida de la variable Entalpía; esto indica, en primer lugar, que la ecuación de continuidad ha sido resuelta satisfactoriamente en los elementos del dominio siendo despreciable la acumulación de masa en el sistema; en segundo lugar estos resultados reflejan una resolución eficiente de la ecuación de conservación de la energía en los volúmenes de control del dominio y un buen modelado del fenómeno de transferencia de calor en el problema simulado. Por el contrario, la variable que presenta mayores residuos finales es la velocidad U para todos los casos; este resultado parece lógico partiendo del hecho de que la velocidad U es la componente de la velocidad que se encuentra en la dirección del flujo y por ende es la que sufre mayores fluctuaciones a lo largo del dominio.

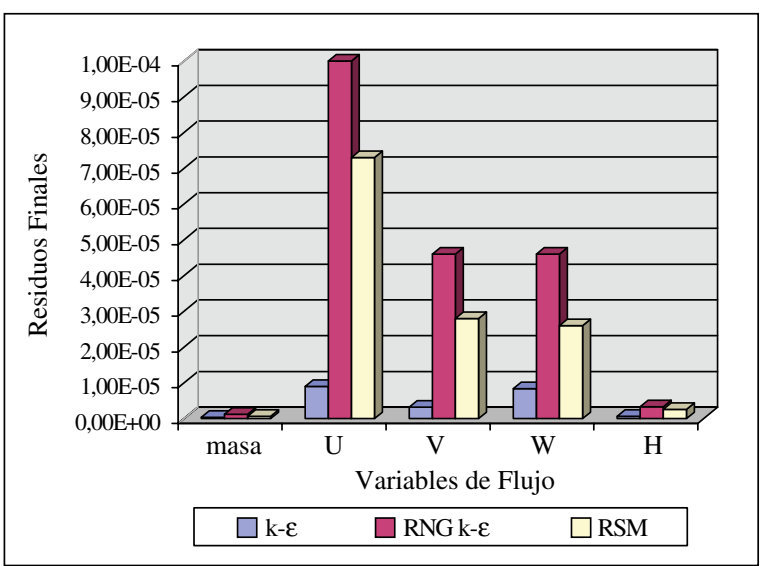

Figura 19. Comparación entre los residuos finales de cada variable.

Los residuos finales de las variables de flujo representados en la figura 19 indican que la aplicación del modelo k- $\varepsilon$ en este sistema permite una solución más exacta de las ecuaciones de gobierno que la resultante de la aplicación del modelo RMS y RNG k- $\varepsilon$, respectivamente.

\section{Análisis de los perfiles de temperatura, presión y velocidad obtenidos en el ducto}

En la sección anterior quedó demostrado que para problema simulado el modelo k- $\varepsilon$ resulta superior desde el punto de vista numérico a los otros modelos de turbulencia empleados, arrojando menores residuos para las variables de flujo en un menor tiempo computacional. Además de comparar los residuos de las variables, se procedió a construir perfiles de temperatura, presión y velocidad del sistema para cada caso simulado con la finalidad de conocer la influencia que tiene esa selección en los resultados obtenidos. Estos perfiles se muestran en las figuras 20, 21 y 22, respectivamente. Para construir estos gráficos se emplearon los valores de las variables temperatura, presión y velocidad, medidos en una línea central a lo largo de ducto.

En cuanto a los perfiles de temperatura (figura 20) se observa prácticamente el mismo comportamiento para los tres casos, con mayores diferencias en la parte final del ducto (zona en la cual se ubican los mayores residuos del dominio). Las diferencias en los valores de temperatura en los diferentes puntos de muestreo (exceptuando el último) se encuentran en el orden de $0,1^{\circ} \mathrm{C}$, valor bastante pequeño que sólo podría ser determinado con un medidor de temperatura muy preciso. Mediante este gráfico se puede observar que la temperatura aumenta en las salidas de aire debido al aumento de presión asociado a este fenómeno y a su vez las mayores temperaturas del dominio están 
ubicadas en la zona final del ducto debido al fenómeno de recirculación del aire presente en esa zona.

Los perfiles de presión (figura 21) muestran un comportamiento similar para los tres casos simulados con diferencias del orden de $2 \mathrm{~Pa}$ para los diferentes puntos de muestreo; esta diferencia representa un valor de $0,2 \mathrm{~mm}$ de columna de agua, medida prácticamente inapreciable en un manómetro de columna de agua que es el instrumento comúnmente empleado para la medición de la presión en este tipo de sistemas. Según se observa en este gráfico, el comportamiento de la presión en el sistema simulado es el siguiente: caída de presión en el tramo previo a la primera salida debido a las pérdidas por fricción que sufre el aire al entrar en contacto con las paredes del ducto, aumento de la presión en las tres salidas debido a la disminución del caudal de aire y presiones más elevadas al final del ducto ocasionadas por el estancamiento del aire.

Finalmente, en la figura 22 está representado el comportamiento de la velocidad del aire en el ducto simulado, observándose disminución de la velocidad en las salidas de aire del sistema, aumento de la velocidad en las reducciones a expensas de la presión y los valores más bajos de velocidad del sistema se observan en la parte final del ducto como consecuencia del estancamiento del aire en esa zona.

Estos gráficos demuestran que para todos los casos simulados el comportamiento de los resultados obtenidos tiene la misma tendencia con diferencias muy pequeñas entre sus valores; por lo tanto seleccionar el modelo de turbulencia k- $\varepsilon$ resulta una selección bastante razonable ya que a pesar de su bajo costo computacional sus resultados son similares a los obtenidos para los otros dos modelos de turbulencia empleados.

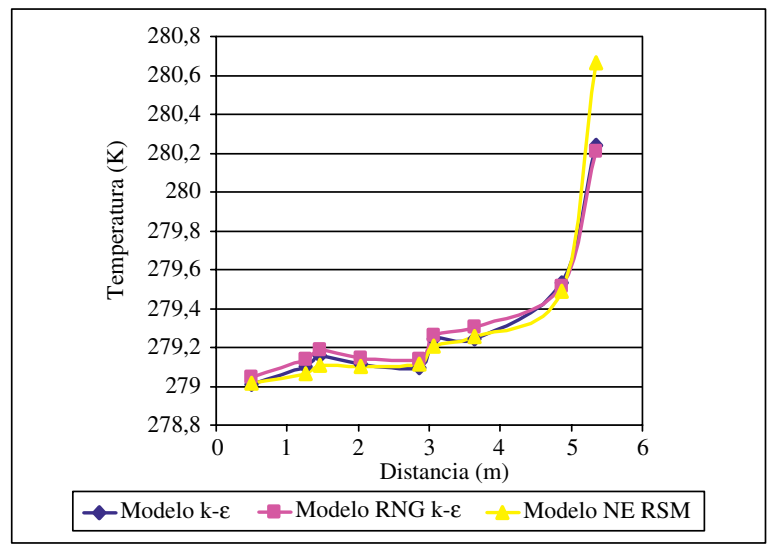

Figura 20. Perfiles de temperatura obtenidos para cada caso simulado.

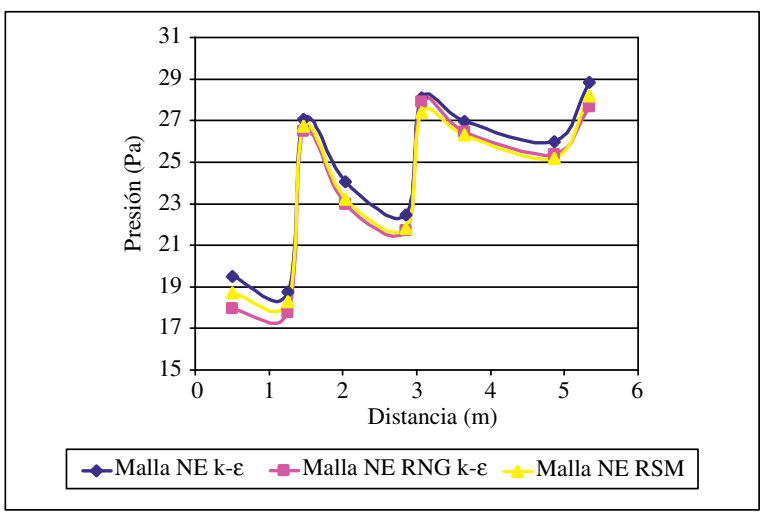

Figura 21. Perfiles de presión obtenidos para cada caso simulado.

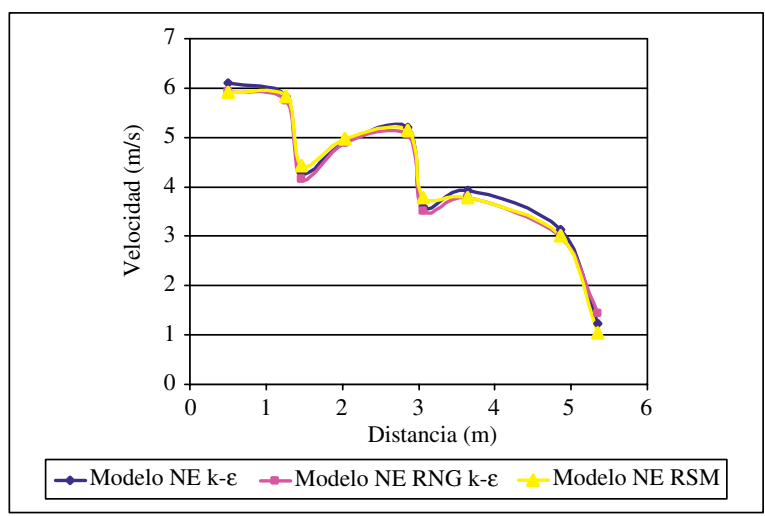

Figura 22. Perfiles de velocidad obtenidos para cada caso simulado.

\section{CONCLUSIONES}

Los resultados obtenidos en el presente trabajo de investigación permitieron establecer las siguientes conclusiones:

1. El modelo matemático empleado en la presente investigación permitió predecir satisfactoriamente el comportamiento termofluidodinámico del aire en el sistema de distribución de aire acondicionado.

2. El modelo de turbulencia k- $\varepsilon$ demostró ser numéricamente superior al modelo RNG k- $\varepsilon$ y al modelo de las tensiones de Reynolds en la predicción del comportamiento del flujo de aire en el sistema de distribución en estudio.

3. El modelo de turbulencia k- $\varepsilon$ arrojó resultados aproximados a los obtenidos con el modelo RNG k- $\varepsilon$ y con el modelo de las tensiones de Reynolds, presentando como ventaja su economía computacional. 
4. Debido a los bajos residuos obtenidos para la masa como variable de flujo para todos los casos simulados, es posible afirmar que en este problema la ecuación de continuidad ha sido resuelta satisfactoriamente en los elementos del dominio y que la acumulación de masa en el sistema es despreciable.

5. Los bajos residuos obtenidos para la entalpía en los tres casos simulados permiten afirmar que la aplicación de los modelos de turbulencia k- $\varepsilon$, RNG k- $\varepsilon$ y el modelo de las tensiones de Reynolds permite una resolución eficiente de la ecuación de conservación de la energía en los volúmenes de control del dominio, representando esto, a su vez, un buen modelado del fenómeno de transferencia de calor en el problema simulado.

6. Por ser la componente de la velocidad U la que sufre mayores fluctuaciones a lo largo del dominio, sus residuos resultan mayores a los de las demás variables del sistema.

7. Los perfiles de velocidad y presión obtenidos para el sistema de distribución simulado muestran un comportamiento que coincide con el señalado teóricamente.

8. La extensión del conducto de distribución luego de la última salida de aire genera una recirculación del flujo que se traduce en mayores pérdidas de carga y en un importante aumento de la temperatura con respecto al resto del dominio.

\section{REFERENCIAS}

[1] A. Valencia y C. Orellana. "Simulación del Flujo Turbulento y la Transferencia de Calor Alrededor de un Cuerpo de Sección Cuadrada". Ciencia Abierta. $N^{o} 7$, p. 5. Universidad de Chile. Santiago, Chile. 2000 .
[2] F. Menter and M Kuntz. "Adaptation of EddyViscosity Turbulence Models to Unsteady Separated Flow Behind Vehicles". Proc. Conf. The Aerodynamics of Heavy Vehicles. Asilomar, Ca. 2002.

[3] J. Shewchuk. "Delaunay refinement algorithms for triangular mesh generation". Computational Geometry: Theory and Applications. 2002.

[4] L. Chen and J. Xu. "Optimal Delaunay Triangulation". Journal of Computational Mathematics. Vol. 22 No 2, pp. 299-308. 2004.

[5] M. Rokni, B. Sunden and L. Davidson. "A Numerical Study of Turbulent Forced Convection in a Square Duct Using Different Turbulence Models". 9th Int. Conf. on Numerical Methods in Thermal Problems, pp. 205-216. Atlanta. 1995.

[6] R. Bird, W. Stewart and E. Lightfoot. "Transport Phenomena". John Wiley \& Sons Inc. Second Edition. New York, EE.UU. 2002.

[7] S. Salamah and D. Kaminski. "Modeling of Turbulent Heat Transfer from an Array of Submerged Jets Impinging on a Solid Surface". Numerical Heat Transfer: Part A: Applications. Vol. $48 \mathrm{~N}^{\circ} 4$. 2005.

[8] Q. Chen and N. Chao. "Comparing turbulence models for buoyant plume and displacement ventilation simulation". Indoor Built Environ. No 6, pp. 140-149. 1997.

[9] Q. Chen and W. Xu. "A zero-equation turbulence model for indoor airflow simulation". Energy and Buildings. Vol. $28 \mathrm{~N}^{\mathrm{o}}$ 2, pp. 137-144. 1998.

[10] Q. Du and D. Wang. "Tetrahedral Mesh Optimization based on CVT"'. Inter. J. Numer. Meth. Eng. Vol. 56 $\mathrm{N}^{\circ}$ 9, pp. 1355-1373. 2002. 